\title{
Mahaim conduction producing left axis deviation and normal QRS
}

HIDEYO MATSUGUCHI, AKIRA TAKESHITA, NAOKI MAKINO, SENICHI
TANAKA, OSAMU NAKAGAKI, AKIO KUROIWA, AND MOTOOMI NAKAMURA

From the Research Institute of Angiocardiology and Cardiovascular Clinic, Kyushu University Medical School, Fukuoka, Fapan

SUMMARY An unusual patient is described in whom electrophysiological studies strongly suggest the occurrence of Mahaim conduction. The patient whose electrocardiogram previously showed a left anterior hemiblock pattern then developed advanced atrioventricular (AV) block (AH block). Beats conducted through the atrioventricular node always had a short $\mathrm{HV}$ interval $(20 \mathrm{~ms})$ and QRS complexes of left anterior hemiblock pattern. Junctional escape beats always had a normal HV interval ( $50 \mathrm{~ms}$ ) with normal intraventricular conduction. His bundle pacing showed the $\mathrm{StV}$ interval and QRS contour of escape beats. These findings suggest the existence of an accessory pathway (Mahaim fibres) passing from the area of block, presumably the uppermost portion of the His bundle, to the posteroinferior division of the left bundle-branch. The surface electrocardiogram did not show the characteristic delta wave of the Wolff-Parkinson-White syndrome. Our observations suggest that patients in whom there is conduction along Mahaim fibres may show only the pattern of intraventricular conduction defect without a delta wave.

Although anatomical studies in cases of the WolffParkinson-White syndrome have occasionally shown the presence of Mahaim fibres (Lev et al., 1975), actual conduction through Mahaim fibres leading to the ventricular pre-excitation is reportedly very rare (Lev et al., 1966; Castillo and Castellanos, 1970; Massumi, 1970; Rosen et al., 1971; Coumel et al., 1972; Castellanos et al., 1975; Lev et al., 1975; Tonkin et al., 1975; Touboul et al., 1975).

Clues to conduction by Mahaim fibres have been classically said to include the presence of a normal or prolonged PR interval with a delta wave (Wolff and White, 1948; Pick and Katz, 1955), and the persistence of pre-excitation in beats of atrioventricular junctional origin (Pick and Katz, 1955; Lev et al., 1966). Recently, His bundle recording with atrial or His bundle pacing has made it possible to recognise Mahaim conduction even in the presence of other accessory pathways such as Kent or James fibres (Coumel et al., 1972; Tonkin et al., 1975).

in the present case, electrophysiological study after the development of advanced atrioventricular

'Present address: The Department of Internal Medicine, University of Iowa Hospitals, Iowa City, IA 52242

Received for publication 27 June 1977 block strongly suggested the existence of Mahaim fibres. Mahaim conduction was associated only with a left anterior hemiblock pattern without a delta wave.

\section{Case report}

A 48-year-old woman was admitted to Kyushu University Hospitals on 12 April 1976. She had had several episodes of palpitation in October 1974, lasting for only 10 seconds or less. An electrocardiogram at that time (Fig. 1, A) showed sinus rhythm with normal $P R$ interval $(0.18 \mathrm{~s})$. The QRS complexes were of normal duration $(0.08 \mathrm{~s})$ without delta wave, but there was left axis deviation $\left(-35^{\circ}\right)$ with $\mathrm{q}$ in aVL and $S$ in lead II and III, compatible with left anterior hemiblock. Six months before admission, she became short of breath on exertion. An electrocardiogram then showed advanced atrioventricular block. She had no syncopal attacks.

On admission, electrocardiograms showed advanced atrioventricular block (Fig. 1, B). The second QRS complex, a conducted beat, shows left axis deviation with QRS duration $0.08 \mathrm{~s}$, similar to that seen in October 1974. Other QRS complexes are junctional escape beats and, in contrast to the 
A

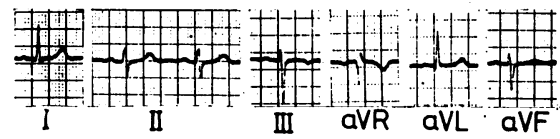

B

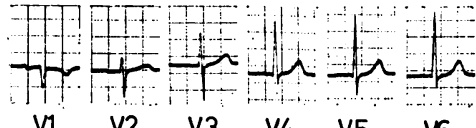

V1 V2 V3 V4 V5 V6
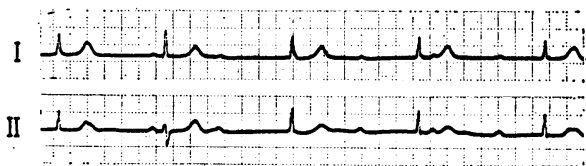

III

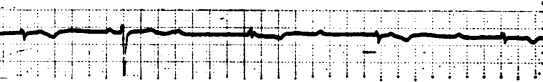

C

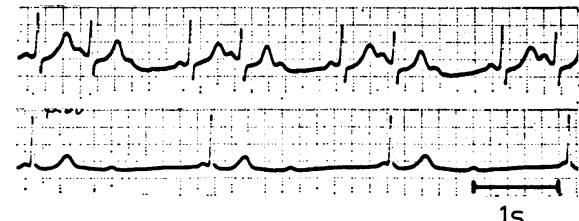

D

is

Fig. 1 (A) Electrocardiogram on 9 October 1974. Normal sinus rhythm $(67 / \mathrm{min})$ with $Q R S$ complexes of left anterior hemiblock pattern.

(B) Electrocardiogram on admission, 13 April 1976. Advanced atrioventricular block is noted. The second $Q R S$ complex, a conducted beat, is similar to those in the upper panel. Other QRS complexes are junctional escape beats and show normal intraventricular conduction.

(C, D) Parts of a 24-hour recording of the electrocardiogram (lead CM 5). (C) Mobitz type II block with 3:2 atrioventricular conduction. The PR interval of conducted beats was constant. (D) functional escape rhythm at 28 beats a minute with normal intraventricular conduction in advanced atrioventricular block. conducted beat, show normal axis $\left(+30^{\circ}\right)$ and the QRS duration $0.06 \mathrm{~s}$. A 24-hour recording of the electrocardiogram (Fig. 1: lower panel) showed advanced atrioventricular block with occasional atrioventricular conduction, mainly $2: 1$, rarely $3: 2$ (Fig. 1, C) or 1:1. The junctional escape rhythm varied from 28 to 44 a minute (Fig. 1, D). When 3:2 atrioventricular conduction was present, the PR interval of the conducted beat was constant, suggesting Mobitz type II block. Every conducted beat showed the left anterior hemiblock pattern, and the same PR interval and QRS duration as in October 1974. During her hospital stay, a repeat 24-hour recording of the electrocardiogram did not show any QRS complex with the delta wave of the classical Wolff-Parkinson-White syndrome.

An electrophysiological study was performed on 21 April 1976. After the study, the patient was treated with oral isoprenaline $(15 \mathrm{mg}$ q.d.) which restored normal $(1: 1)$ atrioventricular conduction. The QRS complexes were similar to those in October 1974, showing left axis deviation.

\section{Electrophysiological study}

The techniques for obtaining His bundle electrograms in our laboratory have been described previously (Takeshita et al., 1974). The best atrioventricular conduction seen during the study was 3:1 conduction with junctional escape beats, as illustrated in Fig. 2. The second and fourth QRS complexes are conducted beats and preceded by the A wave and His bundle deflection. The AH and $\mathrm{HV}$ intervals are $130 \mathrm{~ms}$ and $20 \mathrm{~ms}$, respectively. These QRS complexes show left anterior hemiblock pattern with QRS duration $80 \mathrm{~ms}$. The third A wave is not followed by a His bundle deflection

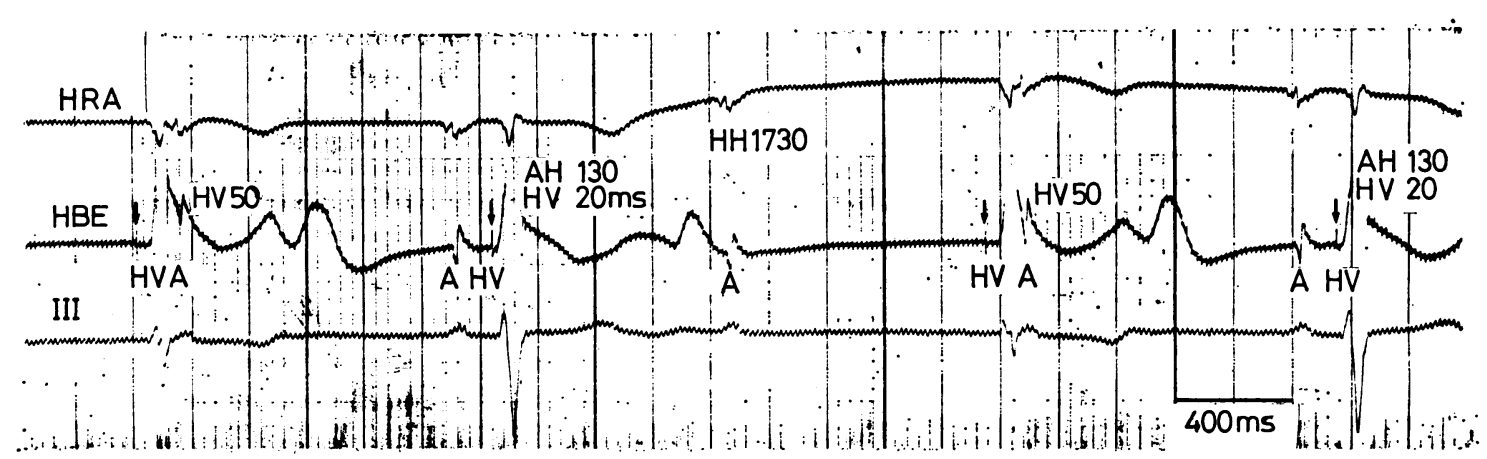

Fig. 2 Recording of the His bundle electrogram $(H B E)$, high right atrial electrogram $(H R A)$, and lead III. The arrow indicates the His deflection. There is 3:1 atrioventricular conduction with junctional escape beats. Conducted beats have normal $A H$, short $H V$ interval $(20 \mathrm{~ms})$, and $Q R S$ complex of the left anterior hemiblock pattern. Functional escape beats have normal $H V$ interval ( $50 \mathrm{~ms}$ ) and $Q R S$ complex shows normal intraventricular conduction. The third $A$ wav is not followed by the His deflection, indicating AH block. 


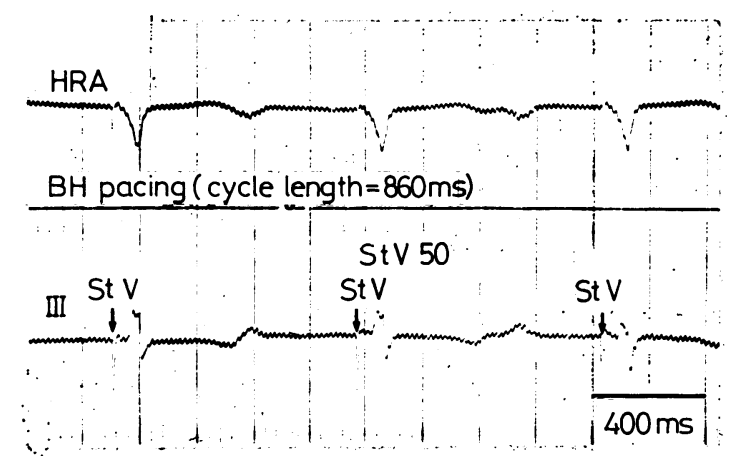

Fig. 3 His bundle stimulation ( $S t$ ) (indicated by arrow) showing that the StV interval and $Q R S$ complex were similar to the $H V$ interval and $Q R S$ complex of junctional escape beats shown in Fig. 2.

indicating $\mathrm{AH}$ block. The first and third QRS complexes are preceded only by the His bundle deflection with $\mathrm{HV}$ interval $50 \mathrm{~ms}$, indicating the origin of these impulses above the bifurcation of the His bundle. The electrical axis of these escape beats is normal and the QRS duration is $60 \mathrm{~ms}$. The conducted beats show a short HV interval (the normal HV interval in our laboratory is 35 to $55 \mathrm{~ms}$ ) but, in contrast, escape beats have a normal HV interval. The QRS duration of conducted beats is longer than that of the junctional escape beats. During the study numerous unsuccessful attempts were made to record a split or other His bundle deflection by varying the position of the His bundle catheter.

Pacing the His bundle (Fig. 3) at a cycle length of $860 \mathrm{~ms}$ (slightly shorter than the spontaneous atrial cycle length) was associated with the stimulus

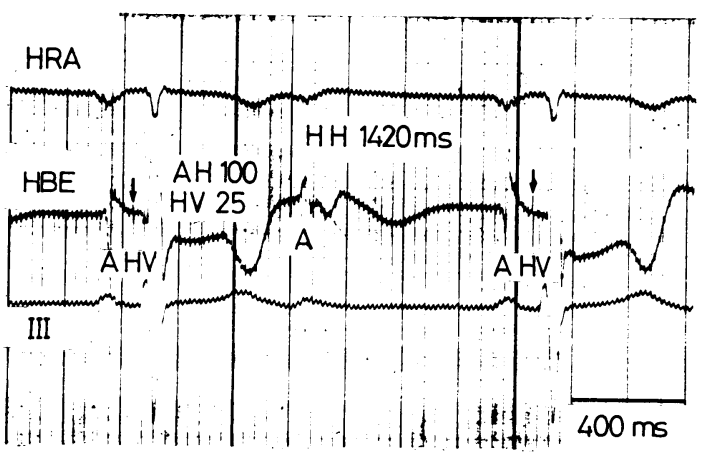

Fig. 4 2:1 atrioventricular conduction after intravenous atropine (1 mg), with short $H V$ interval and $Q R S$ complex of left anterior hemiblock pattern. The absence of junctional escape beats indicates that the cycle length (1730 ms) of junctional escape beats cannot be less than 1420 ms (twice the atrial cycle length). The arrow indicates the His deflection.
H. Matsuguchi et al.

to $\mathrm{V}$ wave (St V) interval of $50 \mathrm{~ms}$ and $1: 1$ con- $c$. duction. The QRS complex with pacing the His $\vec{F}$ bundle was similar to that of escape beats shown in Fig. 2, with electrical axis of $+30^{\circ}$ and the QRS duration $60 \mathrm{~ms}$. The pacing interval was varied between 860 and $660 \mathrm{~ms}$, but the StV interval and the QRS configuration did not change. These findings suggest that intraventricular conduction distal to the His bundle was normal.

Intravenous administration of atropine $(1 \mathrm{mg})$ improved atrioventricular conduction to $2: 1 \mathrm{AH}$ conduction (Fig. 4). Conducted beats showed the QRS complex of left anterior hemiblock pattern with QRS duration $80 \mathrm{~ms}$. As junctional escape beats were not seen after atropine, the junctional escape interval could not have been less than 1420 ms (twice the atrial cycle length).

The slow basic rate of the junctional escape rhythm (28 to 44 per minute) unaffected by atropine indicates that the location of escape pacemaker is in the upper portion of His bundle rather than in
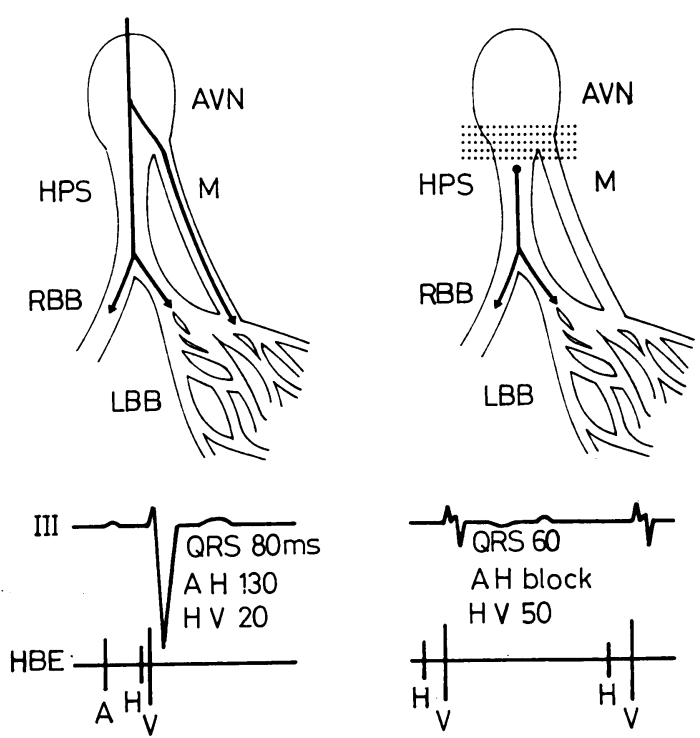

Fig. 5 Schematic illustration of ventricular excitation. The left-hand panel shows a ventricular complex which is composed by fusion of conduction by the normal HisPurkinje system (HPS) and that through Mahaim fibres $(M)$ from the uppermost portion of the His bundle to the posteroinferior divisions of the left bundle-branch $(L B B)$. Pre-excitation of posterobasal endocardial surface of the left ventricle produces a QRS complex of left anterior hemiblock pattern with short $H V$ interval $(20 \mathrm{~ms})$. The right-hand panel shows normal intraventricular conduction of a junctional escape beat with normal $H V$ interval (50 ms), when atrioventricular $(A V)$ block is present. The dotted area indicates simultaneous block both in the normal pathway and Mahaim fibres. 
the atrioventricular node (Narula and Samet, 1971; Scherlag et al., 1973). The presence of Mobitz type II block with 3:2 atrioventricular conduction (Fig. 1, C) indicates that $\mathrm{AH}$ block was in the uppermost portion of the His bundle (Narula et al., 1971).

These finaings may be summarised schematically as shown in Fig. 5. Conducted beats were always associated with a short $\mathrm{HV}$ interval and with a QRS configuration of left anterior hemiblock pattern. Escape beats or His bundle pacing at various rates showed a normal $\mathrm{HV}$ or StV interval and normal electrical axis, indicating normal intraventricular conduction below the His bundle. The degree of shortening of the HV interval in conducted beats was similar to the prolongation of the QRS duration. Though the short HV interval with left axis deviation in conducted beats could conceivably be caused by congenitally short posteroinferior divisions of the left bundle-branch, as postulated in some patients with incomplete endocardial cushion defect (Goodman et al., 1974), this possibility is ruled out by normalisation of the $\mathrm{HV}$ interval and QRS axis in escape beats or during His bundle pacing. Our observations suggest conduction over an accessory pathway in conducted beats, causing pre-excitation of the posterobasal portion of the left ventricle. The atrioventricular block appears to be in the uppermost portion of the His bundle. The observations that conduction in the accessory pathway occurred at the same time as block in the normal atrioventricular conduction pathway, and that retrograde conduction to the accessory pathway did not occur with escape beats or His bundle pacing, suggest that the accessory pathway arises in the region of AH block. The absence of a delta wave on the surface electrocardiogram may be explained by insertion of the accessory pathway into the posteroinferior divisions of the left bundlebranch rather than into the ventricular myocardium as was suggested in the case reported by Tonkin et al. (1975).

\section{Discussion}

The present case is analogous to those reported by Touboul et al. (1975) in which HV intervals were abnormally short at rest, but lengthened suddenly to normal, with disappearance of the pre-excitation pattern during atrial pacing at increasing rates. Normalisation of the $\mathrm{HV}$ interval and QRS pattern was thought to result from functional exclusion of accessory Mahaim conduction. In the present case the observations that conducted beats were associated with a short $\mathrm{HV}$ interval $(20 \mathrm{~ms})$, and that escape beats and His bundle paced beats were associated with a normal $\mathrm{HV}$ interval $(50 \mathrm{~ms})$, can best be explained by intermittent block of Mahaim conduction together with block of the ordinary atrioventricular conduction system in the uppermost part of the His bundle.

The presence of congenitally short posteroinferior divisions of the left bundle-branch as an alternative explanation for the short HV interval with left axis deviation in conducted beats is ruled out by normalisation of the HV interval and QRS axis with junctional escape beats or by His bundle pacing. Another possible explanation for the findings in the present case could be the presence of an area of intermittent block in the His bundle with a localised area of permanent impairment of conduction including those fibres constituting the left anterior division of the left bundle-branch. If this were the case, in order to explain the short HV interval, one would have to postulate that His bundle deflections observed during normal atrioventricular conduction were recorded from the site of delay in the fibres of the left anterior fascicle. However, the failure to record a different His deflection with normal HV interval when attempts were made to do so by varying the position of the His bundle catheter makes this possibility unlikely.

In the present series, Mahaim conduction was associated only with a left anterior hemiblock pattern. Classical Wolff-Parkinson-White complexes were not noted even during repeated 24 hour recordings of the electrocardiogram. Previous reports suggest that Mahaim conduction may be associated with various degrees of the ventricular pre-excitation (Lev et al., 1966; Castillo and Castellanos, 1970; Massumi, 1970; Rosen et al., 1971; Coume! et al., 1972; Castellanos et al., 1975; Lev et al., 1975; Tonkin et al., 1975; Touboul et al., 1975). The classical Wolff-Parkinson-White pattern with the delta wave was seen in cases reported by Lev et al. (1966, 1975), Massumi (1970), Coumel et al. (1972), and Touboul et al. (1975). In some cases, Mahaim conduction may produce slight initial slurring of the QRS complex (Castillo and Castellanos, 1970; Rosen et al., 1971) or only bundle-branch block patterns (Castellanos et al., 1975; Tonkin et al., 1975). It has been suggested that if Mahaim fibres end in the bundle-branch system or close to the Purkinje network, preexcitation may be insufficient to produce a definite delta wave (Castellanos et al., 1975; Tonkin et al., 1975). The left anterior hemiblock pattern seen in conducted beats in the present case may be explained by Mahaim fibres ending in the posteroinferior divisions of the left bundle-branch. This condition may be analogous to that seen in some patients with incomplete endocardial cushion 
defect, in whom a short $\mathrm{HV}$ interval and left anterior hemiblock pattern have been attributed to congenitally short posteroinferior divisions of the left bundle-branch (Goodman et al., 1974). The absence of a right bundle-branch block pattern in the present case is not unexpected; many of the patients with incomplete endocardial cushion defect reported by Goodman et al. had no right bundle-branch block pattern after operation, despite congenitally short posteroinferior divisions of the left bundle-branch and resulting short HV interval and left axis deviation. Our case is the first to be reported in which the left anterior hemiblock pattern appears to be produced by conduction over Mahaim fibres.

\section{References}

Castellanos, A., Agha, A. S., Befeler, B., and Myerberg, R. J. (1975). Double accessory pathways in Wolff-ParkinsonWhite syndrome. Circulation, 51, 1020-1025.

Castillo, C. A., and Castellanos, A., Jr. (1970). His bundle recordings in patients with reciprocating tachycardias and Wolff-Parkinson-White syndrome. Circulation, 42, 271-285.

Coumel, P., Waynberger, M., Fabiato, A., Slama, R., Aigueperse, J., and Bouvrain, Y. (1972). Wolff-ParkinsonWhite syndrome. Problems in evaluation of multiple accessory pathways and surgical therapy. Circulation, 45, 1216-1230.

Goodman, D. J., Harrison, D. C., and Cannom, D. C. (1974). Atrioventricular conduction in patients with incomplete endocardial cushion defect. Circulation, 49, 631-637.

Lev, M., Fox, S. M., Bharati, S., Greenfield, J. C., Jr., Rosen, K. M., and Pick, A. (1975). Mahaim and James fibers as a basis for a unique variety of ventricular preexcitation. American fournal of Cardiology, 36, 880-888.

Lev, M., Leffler, W. B., Langedorf, R., and Pick, A. (1966). Anatomic findings in a case of ventricular pre-excitation (WPW) terminating in complete atrioventricular block. Circulation, 34, 718-733.
Massumi, R. A. (1970). His bundle recordings in bilateral bundle branch block combined with Wolff-ParkinsonWhite syndrome: antegrade type II (Mobitz) block and 1:1 retrograde conduction through the anomalous bundle. Circulation, 42, 287-295.

Narula, O. S., and Samet, P. (1971). Effect of atropine and glucagon on $\mathrm{A}-\mathrm{V}$ nodal and His bundle pacemakers in man (abstract). Circulation, 43 and 44, Suppl. II, 205.

Narula, O. S., Scherlag, B. J., Samet, P., and Javier, R. P. (1971). Atrioventricular block: localisation and classification by His bundle recordings. American fournal of Medicine, 50, 146-165.

Pick, A., and Katz, L. N. (1955). Disturbances of impulse formation and conduction in the pre-excitation (WPW) syndrome-their bearing on its mechanism. American Journal of Medicine, 19, 759-772.

Rosen, K. M., Mehta, A., Rahimtoola, S. H., and Miller, R. A. (1971). Sites of congenital and surgical heart block as defined by His bundle electrocardiography. Circulation, 44, 833-841.

Scherlag, B. J., Lazzara, R., and Helfant, R. H. (1973). Differentiation of 'A-V junctional rhythms'. Circulation, 48, 304-312.

Takeshita, A., Tanaka, S., and Nakamura, M. (1974). Study of retrograde conduction in complete heart block using His bundle recordings. British Heart fournal, 36, 462 467.

Tonkin, A. M., Dugan, F. A., Svenson, R. H., Sealy, W. C., Wallace, A. G., and Gallagher, J. J. (1975). Coexistence of functional Kent and Mahaim-type tracts in the preexcitation syndrome. Demonstration by catheter techniques and epicardial mapping. Circulation, 52, 193-200.

Touboul, P., Huerta, F., Arnaud, P., Porte, J., and Delahaye, J. P. (1975). Etude électrophysiologique de deux cas de préexcitation ventriculaire compatibles avec la présence de fibres de Mahaim (In French). Archives des Maladies $d u$ Coeur et des Vaisseaux, 68, 841-851.

Wolff, L., and White, P. D. (1948). Syndrome of short P-R interval with abnormal QRS complexes and paroxysmal tachycardia. Archives of Internal Medicine, 82, 446-467.

Requests for reprints to Dr Akira Takeshita, Cardiovascular Division, Department of Internal Medicine, University of Iowa Hospitals, Iowa City, Iowa 52242, U.S.A. 